\title{
End-of-life decisions in neonatal care: a conversation analytical study
}

\author{
Neil Marlow (1) ,' Chloe Shaw, ${ }^{1}$ Kat Connabeer, ${ }^{2}$ Narendra Aladangady, ${ }^{3}$ \\ Katie Gallagher (D) , ${ }^{1}$ Paul Drew ${ }^{4}$
}

'Institute for Women's Health, UCL, London, UK

${ }^{2}$ Social Science, Birmingham City University, Birmingham, UK ${ }^{3}$ Neonatal Unit, Homerton University Hospital, London, UK ${ }^{4}$ Department of Language and Linguistic Science, University of York, York, UK

\section{Correspondence to}

Professor Neil Marlow, Institute for Women's Health, UCL, London WC1E 6AU, UK; n.marlow@ucl.ac.uk

Received 2 May 2020

Revised 10 August 2020

Accepted 16 August 2020
ABSTRACT

Objective To understand the dynamics of conversations between neonatologists and parents concerning limitation of life-sustaining treatments.

Design Formal conversations were recorded, transcribed and analysed according to the conventions and methods of conversation analysis.

Setting Two tertiary neonatal intensive care units. Participants Consultant neonatal specialists and families.

Main outcome measures We used conversation analysis and developed an inductive coding scheme for conversations based on the introduction of limiting lifesustaining treatments and on the parental responses.

Results From recordings with 51 families, we identified 27 conversations about limiting life support with 20 families and 14 doctors. Neonatologists adopted three broad strategies: (1) 'recommendations', in which one course of action is presented and explicitly endorsed as the best course of action, (2) a 'single-option choice' format (conditional: referring to a choice that should be made, but without specifying or listing options), and (3) options (where the doctor explicitly refers to or lists options). Our conversation analysis-informed coding scheme was based on the opportunities available for parents to ask questions and assert their preference with minimal interactional constraint or pressure for a certain type of response. Response scores for parents presented with conditional formats ( $n=15$, median 5.0) and options ( $n=10$, median 5.0) were significantly higher than for those parents presented with 'recommendations' ( $n=16$, median 3.75; $p=0.002$ ) and parents were more likely to express preferences $(p=0.005)$.

Conclusion Encouraging different approaches to conversations about limitation of life-supporting treatment may lead to better parent engagement and less misalignment between the conversational partners.

\section{INTRODUCTION}

Introducing the concept of limiting life-sustaining treatment to parents of newborn babies is one of the most challenging conversations in medicine. Such conversations are not uncommon in practice: between $60 \%$ and $80 \%$ of neonatal deaths follow decisions to limit life-sustaining treatment. ${ }^{1}$ For babies born $<27$ weeks of gestation admitted for neonatal intensive care in England during 2006, 76\% of 580 deaths were described as 'planned', and thus followed conversations between neonatologists and parents about limiting treatment. ${ }^{2}$ Such decisions are based rarely on certainty, but rather on considerations of risks of death or survival with significant disability.

\section{What is already known on this topic?}

- A high proportion of neonatal deaths during critical care occur after conversations between doctors and parents about limiting lifesustaining treatment.

- Previous research has used post hoc interviews or questionnaires which may be confounded by the process.

- The trajectory of these conversations has not previously been directly studied.

\section{What this study adds?}

- We recorded interactions about limiting lifesupporting treatment in order to identify three strategies used by doctors in initiating these conversations.

- Providing parents with options for care is more successful in promoting alignment between doctors and parents, compared with use of recommendations or best interest arguments.

The UK Department of Health recommends that 'clinical care decisions, including end-of-life decisions, are made by experienced staff in partnership with the parents'. ${ }^{3}$ Such decisions may be based on concepts of parental authority or medical paternalism, ${ }^{45}$ and can involve 'team recommendations', '(baby's) best interests', 67 among other considerations. Moreover, the opinions of the clinical team may differ. ${ }^{8-10}$ Parents report that they want to be involved in decision-making, but that current involvement is less than optimal, ${ }^{11}$ suggesting that implementation of relevant guidelines is varied. How these various considerations are applied in practice, indeed the process of arriving at lifelimiting decisions, has not previously been studied.

Most research in this area is retrospective, using data from remote interviews ${ }^{12}$ providing insight into the perceptions of doctors and parents. However, such approaches do not help us to understand how decision-making conversations unfold in real time. We therefore undertook a study based on audio and video recordings of end-of-life discussions using conversation analysis (CA) to evaluate technical aspects of decision-making communication between doctors and parents. While others have begun to observe real-time neonatal interactions and code the talk in terms of parent participation in broad terms, ${ }^{13} 14$ our analysis focuses directly on how the design of the doctors' talk facilitates 
parental participation, in terms of what was said and discussed subsequently. Our aim was to determine how doctors engage/ facilitate informed parental involvement in the decision-making process.

\section{PARTICIPANTS AND METHODS}

Families were recruited from two neonatal intensive care units in England (2013-2016). Following informed consent to record conversations from each consultant neonatologist, parents of a critically ill baby, about whom discussions of redirection of care were possible, were recruited. Parents were introduced to the study by their consultant and subsequently recruited by CS or their consultant, who provided further information and obtained informed consent.

\section{Procedure}

Formal conversations between doctors and parents were recorded; a multidisciplinary team including nurses, community nurses and others involved in discharge planning were present on all occasions, but the focus of this study was conversations between doctors and parents. Recordings (made available to the parents in line with recommended practice ${ }^{15}$ ) were anonymised and transcribed in detail. ${ }^{16}$ The method of analysis was CA, a technique widely applied to medical interactions ${ }^{17-19}$ to investigate interaction patterns associated with particular phases or activities (eg, presentations of patients' opening problem, ${ }^{20}$ doctors recommending treatment ${ }^{21}$ ), in order to identify the affordances and limitations of different communication practices. ${ }^{22}$ For the purposes of this study, in accordance with CA studies that have shown, for example, that treatment recommendations take (five) different forms, ${ }^{21}$ those utterances in which critical decisions were presented by doctors were identified, and inspected for their specific linguistic format (grammatical and lexical design). Having identified the different formats in which doctors initiated decisions, we then tracked what implications each of these formats had for parental involvement and response. Parents were not interviewed for this study, which focuses on directly observable conduct, on how interaction works ${ }^{23}$; the possible reasons parents may have had for responding in the way they did were not germane to our study of how they responded (differently) to different decision formats (designs). Non-verbal (embodied) communication was not analysed for the purposes of this part of the study, focusing as it does on the (linguistic) form of doctors' utterances, and parents' (verbal) responses, for example, questions.

A coding framework was devised on the basis of how doctors present decisions to parents (format) and how parents responded (table 1). The quantification of our findings highlights the affordances and implications of different communication practices (delivery formats). Response scores include 'opportunity for further questions' and 'preferences expressed'. Both scores are ordered, with high scores equating to greater parental participation. We summated these scores into an overall 'response score', describing the extent to which different strategies for presenting these decisions related to parental participation in decision-making.

To test the reliability of the coding framework, data were coded by two independent coders. Kappa for the agreement between coders for 'decision format' was 0.80 (95\% CI 0.65 to 0.95 ), indicating 'strong agreement'. ${ }^{24}$ Kappa for aspects of parents' talk was 0.80 (95\% CI 0.65 to 0.95 ) for 'parent questions' (strong agreement), 0.70 (95\% CI 0.52 to 0.87 ) for 'expression of preference' (moderate agreement) and 0.62 (95\% CI 0.46 to 0.78 ) for 'total response score' (moderate agreement). Kappa for other aspects of the doctors' talk when first raising the decision $(n=27)$ was $0.71(95 \%$ CI 0.48 to 0.94$)$ for 'partnership' (substantial agreement) and 0.71 (95\% CI 0.48 to 0.94) for 'Best Interest Score' (moderate agreement). Results were averaged across the two coders. Distributional differences between the presence and absence of identified features of talk for different decision formats were explored using the KruskalWallis test for independent samples or the Mann-Whitney U test for two group comparisons.

\section{RESULTS}

Among the 51 families recruited, we identified 27 conversations (21 families) in which the possibility of limiting life-sustaining treatment was presented to the parents. Cases where the doctor sought 'clarification' of a decision previously made or considered by parents were not included. Two further conversations in which parents had previously expressed strong preferences regarding the continuation of life-sustaining treatment were excluded. From the 20 remaining families and 25 conversations, we identified 41 occasions in which a potential decision about life-sustaining treatment was presented by the doctor. Of those 25 conversations: 11 concerned withdrawal of ongoing life support, including mechanical ventilation; 12 withholding treatment in the event of further deterioration; and 7 'do not resuscitate orders' (five followed the introduction of a decision to withhold or withdraw). ${ }^{25}$ The conversations were led by a total of 14 consultants.

The reasons for considering limiting life-sustaining treatment were: severe perinatal asphyxia $(n=4)$, prematurity with neurological complications $(n=6)$, very premature infant with

Table 1 Conversation analysis-derived coding framework

\begin{tabular}{l} 
Parental responses to the decision \\
\hline Code \\
\hline Opportunity for questions prior to making decision \\
Includes companion questions, parent response precedent. \\
Expression of preference \\
Includes companion responses, parent response precedent. \\
Emotional responses/reactions not included. \\
Lowest score given when multiple responses.
\end{tabular}

Tacit/passive acceptance/implicit resistance (including nodding)/non-concurrence with the 2 doctor's deferral.

Explicit resistance (including negative formulations of the doctor's actions and deferrals 1 mobilised to resist a doctor's recommendation). 
an iatrogenic problem $(\mathrm{n}=1)$, complex congenital (including cardiac) anomalies $(n=8)$ and a threatened preterm delivery of twins at 23 weeks of gestation (one baby with ruptured amniotic membranes) $(n=1)$. The population was typical of the admissions for both neonatal units being of mixed ethnicity (10 Caucasian, 7 Black, 2 'Other' and 1 not recorded) and varied spiritual beliefs ( 9 Christian beliefs, 2 Muslim, 4 none declared and 4 not recorded).

CA revealed that doctors presented the potential decision in three main ways:

1. Recommendations-one course of action is presented and explicitly endorsed as the best course of action $(n=16)$, for example, 'we should change his active intensive care into palliative care.'

2. Single-option choice (conditionals) - referring to a choice that should be made, but without referring to or listing options $(n=15)$. In all cases a conditional form was used, for example, 'should she deteriorate... would you want...'

3. Options-where the doctor explicitly refers to or lists options $(\mathrm{n}=10)$, for example, 'So the options are that we would offer palliative care, which is just comfort and support, or we would offer intensive care.'

(For a detailed analysis of the interactions in which recommendation and option sequences emerged, see Shaw et al. ${ }^{2627}$ )

In 'recommendation' sequences, moving to palliative care was presented and endorsed as the best course of action. Doctors shared evidence, including (frequently) a previously agreed team perspective, supporting the recommendation as a robust conclusion, ${ }^{28}$ rendering any alternative as being a challenge to the doctor or team. We observed that parents either passively accepted the recommendation (with minimal acknowledgement), or frequently responded robustly (eg, 'so you're telling me to kill my baby'), which resulted in derailing the progression of the conversation and misalignment between the doctor and parent(s).

In 'single-option choice' (termed 'conditionals') and 'options' sequences, limiting intensive care was not presented as the only option. In both formats, the baby's 'best interests' tended not to be stated with certainty, and doctors frequently referred explicitly to the need for a joint decision. Doctors thereby acknowledged parents' involvement in the decision without specifying their own perspectives or preferences. Both these strategies enabled parents to assert their preferences freely, and when they asked questions, they were rarely challenging.

All first instances in each conversation of mention of 'best interest' and partnership were coded $(n=50 / 82$; both coders' data cumulated). We found a significant association between the decision format and how best interest was scored $(p<0.001)$. Using the recommendations format $(n=16)$ when the baby's best interests were mentioned, these were presented with certainty. When the conditional $(n=18)$ or options format $(n=16)$ was used, 'best interest' phrasing was overwhelmingly used as a framework to guide decision-making (12/14 cases for conditionals; 12/13 cases for options). A significant association was also found between decision format and partnership score $(p<0.001)$. Using the recommendations format, $13 / 16$ decisions were presented as a medical team decision. In contrast, some conditional $(n=18)$ or option formats $(n=16)$ tended to be presented with an explicit reference to a joint decision (conditionals $n=15$; options $n=7$ ) and others without (conditionals $n=3$; options $n=8$ ), with just one option format ( $\mathrm{n}=0$ for conditionals) being presented as a team decision.

Our coding demonstrates differences in the way parents responded to these alternative formats (figure 1). Total response

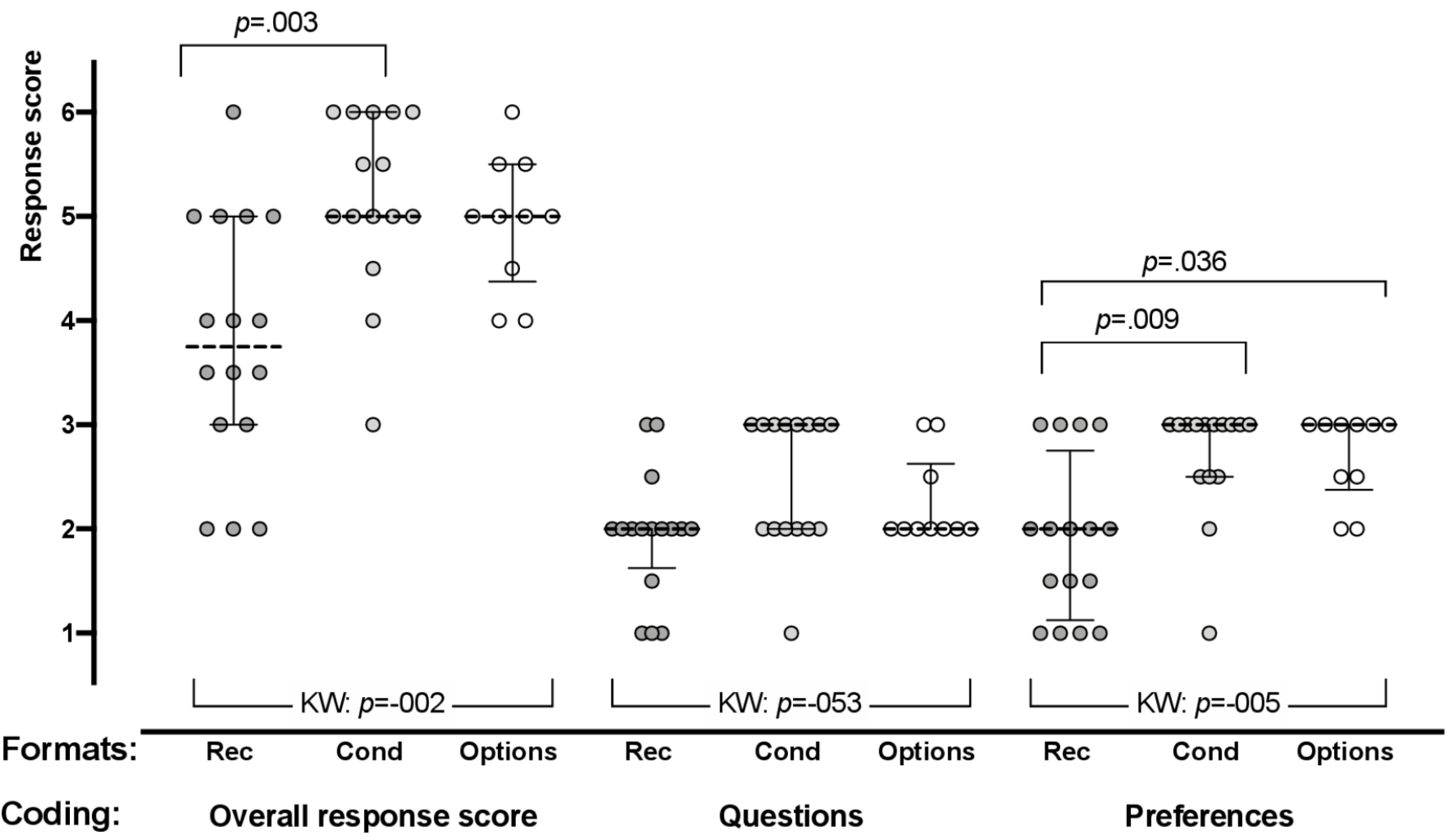

Figure 1 Individual coding scores of different conversational formats for parental responses by asking questions and stating preferences (bars indicate median and quartiles); overall significance shown using Kruskal-Wallis (KW) test and pairwise comparisons after Bonferroni correction. Key to formats: Cond, conditionals; Rec, recommendations. 
Table 2 Median (IQR) for response scores according to first versus subsequent decision points

\begin{tabular}{|c|c|c|c|}
\hline & Questions & Preference & $\begin{array}{l}\text { Total response } \\
\text { score }\end{array}$ \\
\hline \multicolumn{4}{|c|}{ Conversation in a series } \\
\hline First & $2.0(2.0-2.4)$ & $2.5(2.0-3.0)$ & $5.0(4.0-5.0)$ \\
\hline Subsequent & $2.5(2.0-3.0)$ & $3.0(1.5-3.0)$ & $5.0(3.5-6.0)$ \\
\hline \multicolumn{4}{|c|}{ Decision point within a sequence } \\
\hline First & $2.5(2.0-3.0)$ & $2.5(1.5-3.0)$ & $5.0(3.8-5.8)$ \\
\hline Subsequent & $2.0(2.0-2.0)$ & $2.75(2.0-3.0)$ & $4.75(4.0-5.0)$ \\
\hline
\end{tabular}

scores for parents presented with conditionals and options were significantly higher, and parents freely asserted their preference more frequently with both conditional and option approaches compared with recommendation formats. In contrast, the scores allocated for the opportunity for questions did not differ between the three groups, although in the conditional format questions were more often invited or volunteered.

Coding scores did not vary significantly according to whether they were first $(n=28)$ or subsequent $(n=13)$ conversations about reorientation of care in any of the three scales: response scores $(p=0.19)$, questions $(p=0.10)$ or preference $(p=0.59)$. Neither total response nor preference scores differed according to whether they were first $(n=25)$ or subsequent $(n=16)$ mentions within a sequence $(p=0.16$ and $p=0.71$, respectively). First mentions within a sequence, however, scored significantly higher than subsequent mentions for questions $(p=0.017)$ (table 2).

Overall, these results suggest that parental participation was more strongly related to the decision format used by doctors, rather than to any other influencing factors, such as the parent's emotional response or shock at the decision being discussed.

\section{DISCUSSION}

Our study represents a unique approach to evaluating the implications of conversations about end-of-life decisions. We identified three formats through which doctors presented to parents the possible decision to limit life-sustaining treatment: recommendations, conditionals and options. Each led to different conversational trajectories and scales of alignment between parents and doctors. Our findings appear to be statistically robust using our inductive coding, despite the modest number of conversations analysed. One strength of our study lies in the intercoder reliability and therefore the categorisation of the decision formats, for which we developed a CA-informed coding system to support our conclusions. As the first study to use this approach to critical care decision-making in the neonatal intensive care unit, our findings provide evidence that the communication skills of doctors impact on parents' involvement in decision-making-which may further impact parents' ability to cope during the care of their critically unwell baby. This has implications for professional guidance and training in this area.

Conversations using the recommendations format tended to be characterised by reference to a robust, corroborated team decision that should be made in the best interest of the baby. Such a strategy adheres closely to current guidance, yet analysis revealed negative implications for parent participation in the decision-making process, resulting in either misalignment between parties or passive acceptance. Once a recommendation has been stated, the parent is placed in the position of having either to accept or reject the recommendation, with the implication that a rejection challenges medical opinion. Such resistance in response to recommendations has been found elsewhere in decision-making sequences. ${ }^{29} 30$ The use of recommendations invoked little evidence of collaboration, patient-centredness or shared decisions. While this does not preclude the possibility that a recommendation may have a place in these conversations, ${ }^{31} 32$ it should be undertaken in a manner which incorporates, rather than excludes the values of the parents. Offering parental opportunities to discuss the recommendation and ask questions about the rationale behind it may enable parents to engage more fully in the process of decision-making and subsequently.

Conditional and option formats were both characterised by an orientation to joint decision-making between parents and doctors, and provision of information that did not explicitly favour a particular outcome or presuppose the baby's best interests. These formats tended to be followed with stronger alignment between parties where parents were able to assert their preference without misalignment with the doctor and avoided the need for parental acquiescence to professional judgement.

We have previously shown that parent-initiated decision moments (eg, 'so what next') are more likely to be associated with subsequent options from doctors, whereas doctor-initiated decision moments are more likely to be associated with recommendations or conditional formats. ${ }^{27}$ Further research is needed to distinguish the features of conditional formats as compared with options, and the clinical implications of these differences.

Two recent studies of the process of decision-making in endof-life neonatal decision-making have provided a closer examination of these conversations, through analysis of recorded conversations. ${ }^{1314}$ They identified ways in which parent involvement was limited through evidence of minimal psychosocial talk and parent questions in the conversations, ${ }^{13}$ and found little evidence of doctors eliciting parent's preferences. ${ }^{14}$ Although these studies consider parental involvement in a broad sense, in this study we have systematically evaluated parental engagement by recording doctor-parent conversations and analysing how and when talk is produced, moment by moment. Our CA-based coding scheme enables us to analyse decision-making conversations in an inductive manner.

\section{Implications}

It is essential to introduce decision-making in a way that engages parent participation. By exploring options parents may be supported in adjusting to the decision as their own values can be explored and aligned to the decision they make, therefore minimising decisional regret. ${ }^{33}$ This could be important in longterm adjustment to their loss or to the evolution of impairment as their child develops. Even where a baby's death appears to be inevitable, there are choices as to how death can be managed or how a decision on strategy can be reached.

These results have implications for professional training in this area. Previous work has identified that communication skills training is often underserved in neonatology. ${ }^{34} 35$ An educational intervention using role-play and practice exercises with neonatologists in the USA resulted in improvements in doctors' confidence in their ability to communicate prognosis and navigate difficult decision-making with parents. ${ }^{36}$ Further work is required to determine the impact of an educational intervention based on conversation analytical feedback of reallife examples of participants, measured through improved parental engagement in decision-making and professional confidence. 


\section{CONCLUSIONS}

In discussions around the end of life, doctors are professionally enjoined to use recommendations and a test of best interests. ${ }^{67} 37$ The framework as to how best interests are determined remains obscure. ${ }^{38}$ When undertaking challenging conversations with parents, doctors often aim to minimise the burden of guilt they perceive that parents experience. However, Anspach has argued that '...a well-intended but paternalistic attempt to protect parents from guilt may, ironically, produce the very effect it is designed to minimize and may deter, rather than facilitate, vigilant information processing', the latter being a process considered to minimise postdecision regret. ${ }^{5}$ From the work presented here, strategies that encourage true joint decision-making and engage parents may be more effective than the use of recommendations and best interest arguments.

Acknowledgements We acknowledge gratefully the contribution of the parents and neonatologists who allowed us to record and use their personal conversations. We are also grateful for the support and advice of our parent advisory group who reviewed the protocol and parent-facing materials, advised on our approach and the potential for incorporating the findings into training courses and commented on the findings. The group comprised parents who had similar experiences during intensive care and four representatives of interested charities, namely Bliss, Sands, Together for Short Lives and Child Bereavement UK. The group met on five occasions during this study.

Contributors NM and NA conceived the study. NM led the funding application and is the guarantor for the study. All contributors formulated the design and execution of the study. CS and PD carried out conversation analysis, assisted by the clinical members of the team, and analysed the data. CS and KC coded the data. CS and NM wrote the first draft and coordinated the manuscript. All authors were involved in interpretation of the data and writing the report. All have seen and approved the final version

Funding The study was funded by a Programme Development Grant from the National Institute for Health Research and a project grant from Sands. The study was sponsored by University College London. NM receives part funding from the Department of Health's NIHR Biomedical Research Centre's funding scheme at UCLH/UCL.

Disclaimer The funding bodies played no part in the conception, design, analysis or preparation of research outputs.

Competing interests None declared.

Patient consent for publication Not required.

Ethics approval The study received approval from London-City \& East Research Ethics Committee (12/LO/1949).

Provenance and peer review Not commissioned; externally peer reviewed.

Data availability statement Data sharing not applicable as no data sets generated and/or analysed for this study. No data are available for sharing.

\section{ORCID iDs}

Neil Marlow http://orcid.org/0000-0001-5890-2953

Katie Gallagher http://orcid.org/0000-0002-6847-9594

\section{REFERENCES}

1 Confidential Enquiry into Maternal and Child Health (CEMACH). Perinatal Mortality 2007. London: CEMACH, 2009.

2 Costeloe KL, Hennessy EM, Haider S, et al. Short term outcomes after extreme preterm birth in England: comparison of two birth cohorts in 1995 and 2006 (the EPICure studies). BMJ 2012;345:e7976.

3 Department of Health (DoH). Toolkit for High-quality Neonatal Services. London: DoH, 2009

4 Orfali K. Parental role in medical decision-making: fact or fiction? A comparative study of ethical dilemmas in French and American neonatal intensive care units. Soc Sci Med 2004:58:2009-22.

5 Anspach RR. Deciding Who Lives: Fateful Choices in the Intensive-Care Nursery. Berkley. CA: UCP, 1993.

6 General Medical Council (GMC).. Treatment and Ccare Towards the End of Life. London: GMC, 2010.
7 Nuffield Council on Bioethics. Critical care decisions in fetal and neonatal medicine: ethical issues. Report of a Working Group. London: Nuffield Council on Bioethics, 2006.

8 Cuttini M, Nadai M, Kaminski M, et al. End-Of-Life decisions in neonatal intensive care: physicians' self-reported practices in seven European countries. EURONIC Study Group. Lancet 2000:355:2112-8.

9 Gallagher K, Marlow N, Edgley A, et al. The attitudes of neonatal nurses towards extremely preterm infants. J Adv Nurs 2012;68:1768-79.

10 Gallagher K, Aladangady N, Marlow N. The attitudes of neonatologists towards extremely preterm infants: a Q methodological study. Arch Dis Child Fetal Neonatal Ed 2016:101:31-6

11 POPPY Steering Group. Family-centred care in neonatal units. A summary of research results and recommendations from the POPPY project. London: NCT, 2009.

12 McHaffie HE, Laing IA, Parker M, et al. Deciding for imperilled newborns: medical authority or parental autonomy? J Med Ethics 2001:27:104-9.

13 Boss RD, Donohue PK, Larson SM, et al. Family conferences in the neonatal ICU: observation of communication dynamics and contributions. Pediatr Crit Care Med 2016:17:223-30

14 de Vos MA, Bos AP, Plötz FB, et al. Talking with parents about end-of-life decisions for their children. Pediatrics 2015;135:e465-76.

15 Schott J, Henley A, Kohner N. Pregnancy loss and the death of a baby: Guidelines for professionals. 3rd edition. Shepperton, UK: Sands, 2007.

16 Heritage J, Maynard D. Communication in Medical Care: Interactions between Primary Care Physicians and Patients. Cambridge: CUP, 2006.

17 Heritage J, Maynard D. Introduction: Analyzing interaction between doctors and patients in primary care encounters. In: Heritage J, Maynard D, eds. Communication in Medical Care: Interactions between Primary Care Physicians and Patients. Cambridge: CUP, 2006: 1-21.

18 Drew P, Chatwin J, Collins S. Conversation analysis: a method for research into interactions between patients and health-care professionals. Health Expect 2001;4:58-70.

19 Barnes RK. Conversation analysis of communication in medical care: description and beyond. Res Lang Soc Interact 2019;52:300-15.

20 Robinson JD, Heritage J. Physicians' opening questions and patients' satisfaction. Patient Educ Couns 2006:60:279-85.

21 Stivers T, Heritage J, Barnes RK, et al. Treatment recommendations as actions. Health Commun 2018:33:1335-44.

22 Chappell P, Toerien M, Jackson C, et al. Following the patient's orders? recommending vs. offering choice in neurology outpatient consultations. Soc Sci Med 2018;205:8-16.

23 Potter J, Shaw C. The virtues of naturalistic data. In: Flick U, ed. The SAGE Handbook of qualitative data collection. London: Sage, 2017: 182-99.

24 McHugh ML. Interrater reliability: the kappa statistic. Biochem Med 2012;22:276-82

$25 \mathrm{RCPCH}$. Withholding or withdrawing life sustaining treatment in children: A framework for practice. $2^{\text {nd }} e d n$. London: RCPCH, 2004.

26 Shaw C, Stokoe E, Gallagher K, et al. Parental involvement in neonatal critical care decision-making. Sociol Health IIIn 2016;38:1217-42

27 Shaw C, Connabeer K, Drew $\mathrm{P}$, et al. Initiating end-of-life decisions with parents of infants receiving neonatal intensive care. Patient Educ Couns 2020;103:1351-7.

28 Potter J. Representing reality: Discourse, rhetoric and social construction. London: Sage, 1996.

29 Ekberg K, LeCouteur A. Co-implicating and re-shaping clients' suggestions for behavioural change in cognitive behavioural therapy practice. Qual Res Psychol 2014;11:60-77.

30 Quirk A, Chaplin R, Lelliott $\mathrm{P}$, et al. How pressure is applied in shared decisions about antipsychotic medication: a conversation analytic study of psychiatric outpatient consultations. Sociol Health IIIn 2012·34:95-113.

31 Moynihan KM, Jansen MA, Liaw S-N, et al. An ethical claim for providing medical recommendations in pediatric intensive care. Pediatr Crit Care Med 2018:19:e433-7.

32 Toerien M. Deferring the decision point: treatment assertions in neurology outpatient consultations. Health Commun 2018:33:1355-65.

33 Kukora SK, Boss RD. Values-Based shared decision-making in the antenatal period. Semin Fetal Neonatal Med 2018;23:17-24.

34 Gallagher K, Shaw C, Marlow N. Experience of training in communication skills among trainee neonatologists. Arch Dis Child Fetal Neonatal Ed 2015;100:F468

35 Boss RD, Hutton N, Donohue PK, et al. Neonatologist training to guide family decision making for critically ill infants. Arch Pediatr Adolesc Med 2009;163:783-8.

36 Boss RD, Urban A, Barnett MD, et al. Neonatal critical care communication (NC3): training NICU physicians and nurse practitioners. J Perinato/ 2013;33:642-6.

37 Larcher V, Craig F, Bhogal K, et al. Making decisions to limit treatment in life-limiting and life-threatening conditions in children: a framework for practice. Arch Dis Child 2015;100:s1-23.

38 Wilkinson D. Death or Disability?: The 'Carmentis Machine' and decision-making for critically ill children. OUP: Oxford, 2013 